\section{Cartilage integrity and proteoglycan turnover are comparable in canine experimentally induced and human joint degeneration}

\author{
Femke Intema, ${ }^{1}$ Simon Mastbergen, ${ }^{1}$ \\ Cees van Rinsum, ${ }^{2}$ Rene Castelein, ${ }^{2}$ \\ Anne Karien Marijnissen, \\ Johannes Bijlsma, ${ }^{1}$ Floris Lafeber ${ }^{1}$ \\ 'Rheumatology \& Clinical Immunology, \\ University Medical Center Utrecht, \\ The Netherlands; \\ ${ }^{2}$ Orthopaedics, University Medical Center \\ Utrecht, The Netherlands
}

\section{Abstract}

The value of experimental models of osteoarthritis (OA) largely depends on the ability to translate observations to human OA. Surprisingly, direct comparison of characteristics of human and experimental OA is scarce. In the present study, cartilage integrity and matrix turnover in a canine model of joint degeneration were compared to human clinical OA. In 23 Beagle dogs, joint degeneration was induced in one knee, the contra-lateral knee served as a control. For comparison, human osteoarthritic and healthy knee cartilage were obtained at arthroplasty $(n=14)$ and post-mortem $(n=13)$. Cartilage was analyzed by histology and biochemistry. Values for cartilage integrity and proteoglycan (PG) synthesis showed species specific differences; GAG content of healthy cartilage was 2 -fold higher in canine cartilage and PG synthesis even 8 -fold. However, the relative decrease in PG content between healthy and $0 \mathrm{~A}$ cartilage was similar for humans and canines (-17\% vs. $-15 \%$, respectively), as was the histological damage $(+7.0$ vs. +6.1 , respectively) and the increase of PG synthesis $(+100 \%$ vs. $+70 \%$, respectively). Remarkably, the percentage release of total and of newly formed PGs in human and canine controls was similar, as was the increase due to degeneration $(+65 \%$ vs. $+81 \%$ and $+91 \%$ vs. $+52 \%$, respectively). Despite differences in control conditions, the observed changes in characteristics of cartilage integrity and matrix turnover are similar in a canine model of joint degeneration and human clinical OA. The canine Groove model shows that its characteristics reflect those of human $0 \mathrm{~A}$ which makes the model appropriate for studying human $\mathrm{OA}$.

\section{Introduction}

Osteoarthritis (OA) is a highly prevalent degenerative joint disorder. Structural changes primarily comprise degeneration of articular cartilage, accompanied by changes in periarticular bone and synovial inflammation. The disorder develops over years and age and obesity are the main risk factors. ${ }^{1,2}$ Currently, $\mathrm{OA}$ cannot be cured and treatment primarily aims at reducing symptoms (pain) and restoring function. ${ }^{1,2}$ Ideally, disease modifying therapies to prevent progression or to induce repair of joint damage should be applied early in the course of $\mathrm{OA}$. For this reason, studying early changes in the process of $\mathrm{OA}$ is a necessity, as well as studying treatment strategies applied early in the disease. Unfortunately, clinical in vivo studies of early disease have their limitations. Analysis of early (small) cartilage changes (e.g. cartilage matrix integrity and turnover) cannot yet be performed easily by common imaging techniques such as radiographic analysis or MRI. ${ }^{3,4}$ Other non-invasive techniques, like urine and serum biomarker analysis, are still not applicable to monitor changes at an individual level. ${ }^{5}$ Therefore, in vivo animal studies in addition to human in vitro studies are of great value to analyze cartilage at an individual and detailed biochemical level, early in the course of disease.

Animal models have the advantage that the disease can be synchronized and they are relatively quickly progressive. The potential difference in characteristics with human $\mathrm{OA}$ is the main disadvantage. Because the etiology of $\mathrm{OA}$ is poorly understood, it is very hard to measure how well any animal model mimics the disease in humans, even if it may display some features of 0A. The importance of inflammation in $\mathrm{OA}$ is uncertain, as well as the importance of other etiologic factors, such as increase in bone density, and inherited defects in the structure of cartilage collagen. Also major differences among species exist with respect to the relative contribution to pathological changes of various mediators, receptors or enzymes. Nonetheless, models have an important role to play in basic research.

To study the changes associated with cartilage degeneration in $\mathrm{OA}$ in vivo, several animal models of $\mathrm{OA}$ have been developed. Models in horses, goats, sheep, dogs, guinea pigs, rats and mice have been applied (bio)chemically, mechanically, surgically, genetically, etc. ${ }^{6}{ }^{7}$ Most of these models have been validated in such a way that degenerative changes in cartilage matrix occur. Sometimes they have demonstrated to be progressive over time, to be accompanied by intra-articular bone changes, and to have more or less synovial inflammation.

One of the most frequently used joints for a model of $\mathrm{OA}$ is the canine knee joint.
Correspondence: Floris Lafeber, University Medical Center Utrecht, F02.127 P.0. Box 85500, 3508 GA UTRECHT, The Netherlands.

E-mail: f.lafeber@umcutrecht.nl

Key words: osteoarthritis, chondrocyte, experimental model, canine.

Acknowledgements: this study was financially supported by the Dutch Arthritis Association.

Contributions: FI acquisition of data, or analysis and interpretation of data; drafting the article; final approval of the version to be published; SM acquisition of data, or analysis and interpretation of data; drafting the article; final approval of the version to be published; CVR acquisition of human samples; revising the manuscript critically for important intellectual content; and final approval of the version to be published; $\mathrm{RC}$ acquisition of human samples, revising the manuscript critically for important intellectual content; and final approval of the version to be published; AKM conception and design of the study; drafting the article; final approval of the version to be published; JB conception and design of the study; revising the manuscript critically for important intellectual content; and final approval of the version to be published; FL conception and design of the study; revising the manuscript critically for important intellectual content; and final approval of the version to be published.

Conflict of interest: the authors report no conflicts of interest.

Received for publication: 5 May 2010

Revision received: 3 October 2010 .

Accepted for publication: 4 October 2010 .

This work is licensed under a Creative Commons Attribution 3.0 License (by-nc 3.0).

(C) Copyright F. Intema et al., 2010

Licensee PAGEPress, Italy

Rheumatology Reports 2010; 2:e7

doi:10.4081/rr.2010.e7

Arguments in favour of the use of canine (stifle) knee joints are the presence of spontaneously occurring $\mathrm{OA}$ in dogs with a pathology and pathogenesis comparable to human $\mathrm{OA}$, a relative good matching anatomy with the human knee joint, and not unimportantly, size allowing a broad spectrum of analysis and (surgical) interventions.

Recently, in succession to the anterior cruciate ligament transaction (ACLT) ${ }^{8,9}$ and meniscectomy model, ${ }^{10}$ the canine Groove model of joint degeneration was developed. ${ }^{11-13}$ Features of $\mathrm{OA}$ were induced by surgically applied grooves in the weight bearing cartilage of both femoral condyles without damaging the subchondral bone. The contra-lateral knee was validated to be useful as an internal control. ${ }^{11} \mathrm{~A}$ 
bilateral model with a bilateral sham surgery group was validated as well. ${ }^{14}$ The model showed very consistent changes in cartilage bio- and histochemistry in all animals, very similar to those seen in the ACLT model. ${ }^{11}$

While the main goal of experimental models is extrapolation of results to the human pathology, hardly any study has focused on directly comparing cartilage integrity and in particular matrix turnover to human cartilage. When studying treatment strategies, repair capacity of the tissue is expected to be closely related to matrix turnover among other important features such as normalization of intra-articular stress and movement of the joint. Therefore, in the present study we directly compared parameters of cartilage integrity and matrix turnover as observed in the canine Groove model to those observed in clinical human OA.

\section{Materials and Methods}

\section{Canine experimentally induced joint degeneration}

Twenty-three female Beagle dogs, skeletally mature, aged $2.6 \pm 0.4$ years, weighing $11.9 \pm 0.2$ $\mathrm{kg}$, were obtained from the animal laboratory of the Utrecht University, the Netherlands. They were housed in groups of 2-3 dogs per pen, and were let out on a patio in large groups for at least 2 hours daily. They were fed a standard diet and had water ad libitum. For this study, ethical approval was given by the Utrecht University Ethical Committee for animal studies.

Induction of $\mathrm{OA}$ was performed as described previously. ${ }^{11,12}$ In short, surgery was carried out in the right knee through a 2-3 cm medial incision while care was taken to prevent bleeding and soft tissue damage as much as possible. The cartilage of both femoral condyles was damaged by making approximately 10 diagonal grooves to a depth of maximum $0.5 \mathrm{~mm}$ on the weight-bearing area preventing subchondral bone damage. ${ }^{15}$ The latter was checked by histology at the end of the experiment. There was no absolute visual control over the procedure, but macroscopic evaluation after termination of the animals showed similar patterns in all knees. After surgery, synovium, fasciae and skin were sutured. The contra-lateral left unoperated knee served as a control. Starting 2 days after surgery, the dogs were let out on the patio to exercise, 5 days a week for 4 hours a day. The dogs were forced to load the affected joint by fixing the contra-lateral limb to the trunk, 3 days a week for approximately 4 hours a day for 10 weeks.

At 20 weeks post-surgery the dogs were killed with an intravenous injection of Euthasate. Control and degenerated joints were amputated and within 2 hours full thickness cartilage slices were collected from the weight-bearing area of both condyles of both knees and processed under laminar flow conditions.

\section{Human joint degeneration}

Human degenerated cartilage was obtained from the weight bearing area of the femoral condyles during joint replacement surgery from 14 patients with clinically defined $\mathrm{OA}$ (mean age $67 \pm 3$ years; male-female 6:8). It should be kept in mind that only the cartilage that could be cut from the joint surfaces after replacement surgery was used. Similar, from 13 donors intact healthy femoral knee cartilage was obtained from the weight-bearing area post-mortem (mean age $66 \pm 5$ years; malefemale 5:8). Macroscopically, the cartilage had a smooth glossy appearance without any signs of degeneration, which is been confirmed by histology. ${ }^{16}$ Full thickness cartilage slices of both condyles were kept in phosphate buffered saline and were processed under laminar flow conditions within 2 hours after collection. For this study, ethical approval was given by the Medical Ethical Review Committee of the Utrecht Medical Center Utrecht.

\section{Cartilage analysis}

From each joint, full thickness cartilage slices were cut into square full-thickness pieces and weighed (5-15 mg; accuracy $0.1 \mathrm{mg}$ ). For histology, four samples randomly taken from the femoral condyles were fixed in $4 \%$ phosphate-buffered formalin containing $2 \%$ sucrose ( $\mathrm{pH}$ 7.0). Cartilage degeneration was evaluated in safranin-0 fast-green iron hematoxylin stained sections by light microscopy according to the OARSI scale for canine experimental models of $\mathrm{OA}$, a new scoring method for cartilage and bone, initiated by the Osteoarthritis Research Society International (OARSI) and developed by experts in the field. ${ }^{17}$ The scale is based on three categories (similar to the Mankin scale), cartilage surface, cellular characteristics and reduction of safranin-0 staining (indicating loss of proteoglycans). Each category has a maximum score of twelve dependent on the severity and extent of the features, bringing the overall maximum score to thirty-six. Specimens were graded in random order by an observer unaware of the source of the cartilage. The mean score of the four samples was used for statistical evaluation.

For biochemical analysis the cartilage samples (at least 6 for each dog joint and 10 for each human joint) were cultured individually according to standard procedures as described previously $^{18}$ and cartilage proteoglycan (PG) content, PG release, PG synthesis, and release of newly formed PG were determined.

Proteoglycan (PG) content and turnover have been analyzed as described before, ${ }^{11,18}$
Cartilage was cut as thick as possible while excluding the underlying bone. Samples were cut into full-thickness cubes, kept in $200 \mu \mathrm{l}$ culture medium (Dulbecco's Modified Eagle's Medium (DMEM) supplemented with 0.085 $\mathrm{mM}$ ascorbic acid, $2 \mathrm{mM}$ glutamine, $100 \mathrm{IU} / \mathrm{mL}$ penicillin, $100 \mathrm{mg} / \mathrm{mL}$ streptomycin, and $10 \%$ heat-inactivated Beagle serum or human serum) and weighed (5-10 mg; accuracy 0.1 $\mathrm{mg}$ ). Cartilage samples were cultured individually in 96 well culture plates (NUNCLON Denmark) in $200 \mu \mathrm{L}$ culture medium.

Proteoglycan synthesis and newly formed release was determined by exposing the cartilage samples to radioactive sulphate for 4 hours. After subsequent thorough rinsing the samples were cultured in medium without sulphate label for an additional 3 days. Thereafter, medium was collected and the cartilage tissue samples were digested with papain. The amount of ${ }^{35} \mathrm{SO}_{4}{ }^{2}$-labelled GAGs in the papain digest and medium was measured by liquid scintillation analysis and normalized to labelling time and wet weight of the explants. Total PG synthesis is the sum of the labelled GAGS from the papain digest and medium and was expressed as the amount of sulphate incorporated per hour per gram wet weight of the cartilage (nmol/h.g). Release of newly formed PGs (in the medium in 3 days) was expressed as percentage of the originally synthesized (labelled) PGs.

Proteoglycan content and total release was determined by precipitating and staining the GAGs in the papain digest and in the 3-day culture medium with Alcian Blue dye solution. Blue staining of the digested cartilage and the 3-day medium was quantified photometrically with chondroitin sulphate (Sigma C4384) as a reference. The GAG content is the sum of the content in papain digest and medium, corrected for wet weight of the cartilage tissue $(\mathrm{mg} / \mathrm{g})$. The amount of GAGs released to the medium from the matrix is expressed as percentage of total GAG content (percentage release).

\section{Calculations and statistical analysis}

Mean values of different animals or donors ( $\mathrm{n}=23$ degenerated and 23 (contra-lateral) control animal joints, $n=13$ human healthy cartilage donors and $n=14$ human 0 A cartilage donors) \pm SEM are presented. Unpaired nonparametric statistical evaluation was used (Mann-Whitney U test) for optimal comparisons for both animal and human cartilage. Additionally, the percentage or absolute (histology) difference between the degenerated cartilage samples was compared to the mean values of control canine and human cartilage. As such the change between control and degenerated cartilage could be compared between canines and humans by use of MannWhitney U test. 


\section{Results}

\section{Characteristics of control cartilage}

Histological grading according to the newly developed OARSI scale showed an average score $( \pm \mathrm{SEM})$ of $7.2 \pm 1.3$ for human control cartilage and $2.4 \pm 0.5$ for canine control cartilage. This difference between human and canine controls was statistically significant $(\mathrm{P}<0.05)$. Also control values of $\mathrm{PG}$-content and PG synthesis rate were different for both species $(20.9 \pm 2.1$ vs. $45.1 \pm 2.0, \mathrm{P}<0.01$ and $1.1 \pm 0.2$ vs. $8.2 \pm 0.6, \mathrm{P}<0.05$, for human vs. canine control cartilage, respectively). On the other hand, percentage release of total PGs from the matrix during 3 days of culture was similar for human and canine control cartilage ( $7.4 \pm 0.6$ and $6.2 \pm 0.5$, respectively, ns). Also percentage release of newly synthesized PGs during 3 days is similar for human and canine control cartilage ( $8.4 \pm 0.4$ and $8.8 \pm 0.4$, respectively, ns).

\section{Changes in characteristics of degenerated compared to control cartilage}

Representative micrographs are depicted in Figure 1. The degenerated cartilage of the experimental canine joints was on average graded $8.5 \pm 0.9$, which is indicative of mild damage (Figure 1E), statistically significantly higher than that of the control cartilage. Histological damage in human degenerated cartilage as compared to human control cartilage was also observed with an average grade of $14.2 \pm 1.0$, representing moderate cartilage damage. It should be kept in mind that only the cartilage that could be cut from the joint surfaces after replacement surgery was used. Thus in fact the entire joint had a worse appearance than represented by the score of the cartilage used. The mean change in OARSI grade in the canine degenerated cartilage (+6.1) was not statistically significantly different compared to human osteoarthritic cartilage (+7.0). In addition, only minor differences were observed in the contribution of each of the categories to the total score. Cellular characteristics had exactly the same contribution (37\% in both species), while cartilage surface damage (37\% in human $0 \mathrm{~A}$ vs. $32 \%$ in canine degeneration) and loss of staining ( $26 \%$ vs. $31 \%$, respectively) differed insignificantly.

The microscopically observed loss of Safranin-0 staining was supported by the biochemical analysis of the cartilage. In both human $\mathrm{OA}$ and canine experimental cartilage a statistically significant lower PG content was observed (Figure 2A). Despite the fact that the PG content of canine control cartilage was twice as high as that of healthy human carti-
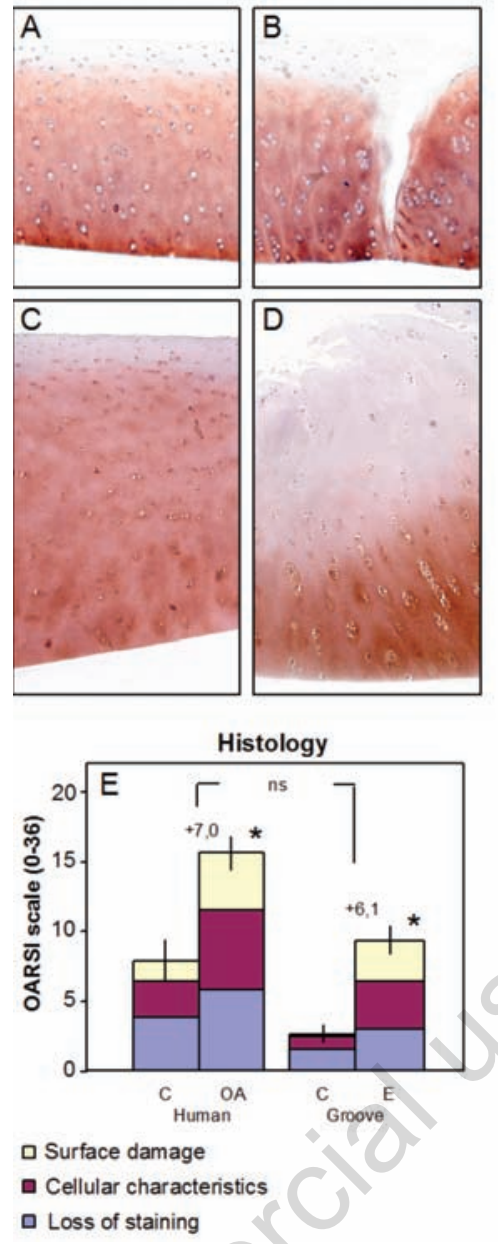

Figure 1. Representative photographs of the control and experimental cartilage of the canine Groove model $(\mathrm{A} / \mathrm{B})$ as well as human healthy and OA cartilage (C/D) are given, safranin- $O$ fast-green iron hematoxylin staining. Figure 1E represents histological grading of osteoarthritis (OA) according to the OARSI scale for experimental models (0-36). An asterisk indicates statistical significant differences compared to control cartilage, $P<0.05$. The changes (control vs. OA/degenerated) between human and experimental OA cartilage were not statistically significantly different (ns).

lage, the relative difference of PG content in both human $0 A$ cartilage and the experimental canine cartilage was comparable (-17\% vs. $15 \%$, respectively).

The lower PG content is the result of an increased loss of PGs from the cartilage matrix. When measuring PGs released from the matrix over a period of three days of both human $\mathrm{OA}$ and canine degenerated cartilage, there was a statistically significantly higher release (Figure 2B) compared to controls. The percentage change was comparable for both species.

To compensate for this increased release there is an increased synthesis of proteoglycans. Synthesis rate of PGs (depicted in Figure 2C) was on average almost twice as high in the experimental joints of the canine model com-
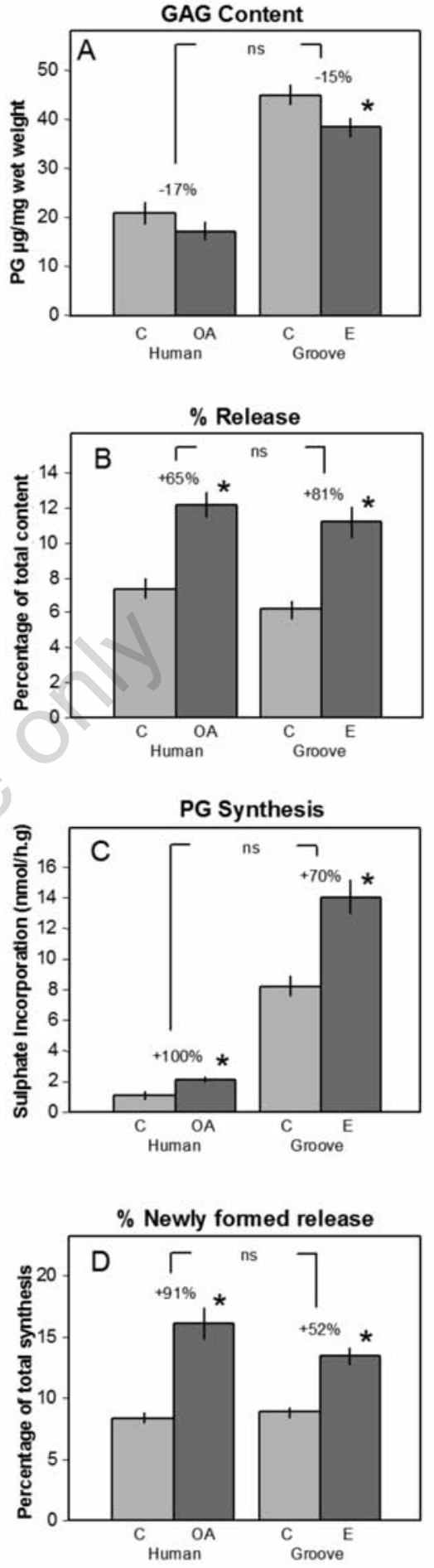

Figure 2. A, PG content (mg/g wet weight cartilage), Percentage release of total PG content (B), PG synthesis rate (C) and percentage release of total newly formed PGs are shown. The absolute differences between control $(C)$ and $O A$ or experimental (E) cartilage are given for human OA and the canine groove model, respectively. An asterisk indicates statistical significant differences compared to control cartilage, $\mathbf{P}<\mathbf{0 . 0 5}$. The changes (control vs. OA/degenerated) between human and experimental OA cartilage were not statistically significantly different (ns). 
pared to the contra-lateral control joints, very similar to the doubling of PG synthesis rate as seen in the OA compared to healthy human cartilage. This higher synthesis of $\mathrm{PGs}$ is largely ineffective since the percentage release of these newly formed PGs is also higher in the osteoarthritic human knee joints compared to human healthy control joints (Figure 2D). Again, this enhanced release of newly formed PGs is also seen in the canine experimental joints, not statistically different from human clinical $0 A$.

There was no statistical significant change in cartilage DNA content due to induction of OA (OA: $0.14 \pm 0.04 \mathrm{mg} / \mathrm{g}$ vs. control: $0.18 \pm 0.07$ $\mathrm{mg} / \mathrm{g}$ and $0.15 \pm 0.04 \mathrm{mg} / \mathrm{g}$ vs. $0.17 \pm 0.04 \mathrm{mg} / \mathrm{g}$, for femoral cartilage of canines and humans respectively, data not shown).

\section{Discussion}

Generally appreciated, but for the first time demonstrated by direct comparison, canine and human cartilage differ significantly with respect to parameters of proteoglycan content and turnover. Irrespectively, changes due to experimentally induced joint degeneration or osteoarthritis in the canine and human cartilage, respectively, are very similar.

In most animal in vivo studies on osteoarthritis, species differences in cartilage structure and chondrocyte activity are either accepted or ignored. However, we hardly have any knowledge on how these differences are of influence on development of osteoarthritis in a quantitative and qualitative way, and even less on how efficacy of treatment modalities is influenced by these differences. Actually, we even have limited knowledge of the exact differences in characteristics of healthy cartilage.

When we look at cartilage structure, thickness of the hyaline cartilage layer in both humans and canines seem to depend on species size, approximately $2.3 \mathrm{~mm}$ for human femoral condyles and $0.8 \mathrm{~mm}$ for canine condyles. ${ }^{15,19}$ As for chondrocyte density, there is an opposite relationship between cartilage thickness and cell density, resulting in a fourfold increase in cell density in canines compared to humans. ${ }^{19,20}$ The specific activity of chondrocytes from different species can also be variable. ${ }^{21}$ In our study it appeared that proteoglycan synthesis as measured by ${ }^{35} \mathrm{SO}_{4}$ incorporation in Alcian Blue precipitated GAGs upon papain digestion is eight fold higher in canines compared to humans. It is tempting to suggest that taking the four fold chondrocyte number into account the canine cells have a 2 fold higher proteoglycan synthesis rate. However, it should be kept in mind that matrix composition (characteristics) could be of influence on digestion and precipitation procedures and with that outcome in absolute numbers.

In our study the PG content as measured by Alcian Blue staining and precipitation in healthy canines is higher than in humans. The higher PG content is expressed per wet weight. As such, the difference might be explained by a lesser capacity to absorb/retain water in the matrix. A species specific artefact due to swelling as a result of collagen damage at the cutting edges might be responsible. Thinner canine cartilage of comparable weight as thicker human cartilage has a different surface/volume ratio, due to the different shape of samples. In addition, qualitative differences in proteoglycan composition between both species can be of influence. Such differences might result in differences in precipitation and staining abilities.

Separated analyses of cartilage sampled from areas with lesions vs. non-lesion areas might give more information regarding differences between degenerated and healthy cartilage in both species. However, there is a rather large variation in the lesions occurring, especially in the human OA samples.

Also the age of the animals differs from the human sample population. Articular cartilage aging changes that may lead to articular cartilage degeneration include fraying and softening of the articular surface, decreased size and aggregation of proteoglycan aggrecans and loss of matrix tensile strength and stiffness. These changes most likely are the result of an age related decrease in the ability of chondrocytes to maintain and repair the tissue. ${ }^{22}$

However, irrespective of all these potential differences, the evaluated characteristics are in a comparable order of magnitude in both species. This is a major advantage over smaller animals used for OA models. In rats and mice the cellularity increases a 26 - and 33 -fold, respectively. ${ }^{19}$ Cartilage thickness is only 0.06 $0.07 \mathrm{~mm} .{ }^{19}$ For these smaller species, to our knowledge there are no data of biochemically determined proteoglycan content or synthesis rate, expressed in such a way that they can be compared to human data.

Osteoarthritis is a disease which not solely affects cartilage. Indeed other tissues are involved as well, such as subchondral bone, synovial tissue among others. Especially the role of subchondral bone in OA has gained a lot of interest the last years. A lot of new knowledge has come forward, but whether subchondral bone changes occur first, later or at the same time is still subject of discussion. However, in the present study there was a focus on proteoglycan turnover of cartilage. Also with respect to cartilage itself many other parameters could have been analyzed. We primarily wanted to show that evaluation of proteoglycan turnover in the canine model of $\mathrm{OA}$ might be of relevance to proteoglycan turnover in human OA. For other parameters of carti- lage and for other tissues such a comparison would also be of value.

The pathogenesis of human $\mathrm{OA}$ is not completely understood. Osteoarthritis is a group of overlapping presumably distinct diseases, with different aetiologies, but with general similar biologic, morphologic and clinical outcome..$^{23}$ Indeed, irrespective of the (unknown) mechanism(s) responsible for OA development, in all of the 14 cases of $\mathrm{OA}$ in this study, cartilage changes with respect to the parameters evaluated were in the same order. In the present study we have chosen for a comparison of characteristics of human OA cartilage with presumably different aetiology with those of degenerated cartilage as observed in the canine Groove model OA with a single aetiology. Although the model has its specific characteristics with respect to induction (aetiology) of cartilage degeneration, it has been described that the degenerative changes are very similar to those seen in other models like the ACLT model of OA. ${ }^{11}$ This fits the idea that also in the canine knee joint different aetiologies e.g. cartilage damage as in the Groove model and joint instability as in the ACLT model, lead to similar biologic and morphologic outcome. An identical similarity of human OA cartilage with canine degenerated cartilage due to joint instability would have been found.

The comparable changes due to joint degeneration in both species with respect to cartilage integrity and turnover, show us that despite basic differences, an experimental model for a complex disorder like OA might be suitable to study OA in many aspects.

However, we still have to guess about the effect of the observed basic differences in cartilage structure and turnover on attempts to remodel or even repair the tissue. Although the degenerative changes with respect to proteoglycan turnover are comparable for canine degenerated and human OA cartilage, differences may not be ignored when this and other models are used to study treatment modalities. It may well be that the turnover rate (under control conditions 8 fold higher) in canine cartilage may demonstrate treatment effects that will take a much longer time span to lead to actual changes in matrix integrity in humans. In general, this may be a major flaw of animal models representing a chronic very slowly developing disease as $0 \mathrm{~A}$. The smaller the species used, the larger the potential difference to humans since slow development is expected to be related to slow cure, assuming cure is possible. Good results of treatment modalities in smaller models might need much longer treatment periods in larger models or may never be found.

Although not of primary relevance to the present study it should be mentioned that canine models of OA have the advantage over most other animal models that force plate 
analyses provide a surrogate but objective parameter for pain/disability of each paw. ${ }^{24}$ In addition to the similarities between cartilage proteoglycan parameters in both models, this gives canine models of $\mathrm{OA}$ a significant advantage over many other models.

In conclusion, animal models are an essential part of research on $\mathrm{OA}$, aiming at better understanding the pathophysiology of $\mathrm{OA}$, especially in its early phases, and to study effects and mechanisms of treatment. Despite species-specific differences in cartilage characteristics in human $\mathrm{OA}$ and canine joint degeneration, similarities in changes due to the degenerative process prevail.

\section{References}

1. Buckwalter JA, Mankin HJ. Articular cartilage: degeneration and osteoarthritis, repair, regeneration, and transplantation. Instructional course lectures 1998;47:487504.

2. Moskowitz RW. Osteoarthritis. Fourth ed. Philadelphia: Lippincott Williams \& Wilkins, a Wolters Kluwer Business; 2007.

3. Eckstein F, Burstein D, Link TM. Quantitative MRI of cartilage and bone: degenerative changes in osteoarthritis. NMR in biomedicine 2006;19:822-54.

4. Eckstein F, Mosher T, Hunter D. Imaging of knee osteoarthritis: data beyond the beauty. Curr Opin Rheumatol 2007;19:435-43.

5. Rousseau JC, Delmas PD. Biological markers in osteoarthritis. Nature clinical practice. 2007;3:346-56.

6. van den Berg WB. Lessons from animal models of osteoarthritis. Curr Opin Rheumatol 2001;13:452-6.

7. Ameye LG, Young MF. Animal models of osteoarthritis: lessons learned while seeking the "Holy Grail". Curr Opin Rheumatol 2006;18:537-47.
8. Brandt KD, Myers SL, Burr D, Albrecht M. Osteoarthritic changes in canine articular cartilage, subchondral bone, and synovium fifty-four months after transection of the anterior cruciate ligament. Arthritis Rheum 1991;34:1560-70.

9. Pond MJ, Nuki G. Experimentally-induced osteoarthritis in the dog. Ann Rheum Dis 1973;32:387-8.

10. Moskowitz RW, Howell DS, Goldberg VM, et al. Cartilage proteoglycan alterations in an experimentally induced model of rabbit osteoarthritis. Arthritis Rheum 1979;22: $155-63$.

11. Marijnissen AC, van Roermund PM, TeKoppele JM, et al. The canine 'groove' model, compared with the ACLT model of osteoarthritis. Osteoarthritis and cartilage/OARS, 0steoarthritis Research Society 2002;10:145-55.

12. Marijnissen AC, van Roermund PM, Verzijl $\mathrm{N}$, et al. Steady progression of osteoarthritic features in the canine groove model. Osteoarthritis and cartilage/0ARS, Osteoarthritis Research Society 2002;10: 282-9.

13. Mastbergen SC, Marijnissen AC, Vianen $\mathrm{ME}$, et al. The canine groove model of osteoarthritis is more than simply the expression of surgically applied damage. Osteoarthritis and cartilage/OARS, Osteoarthritis Research Society 2006;14: 39-46.

14. Intema F, DeGroot J, Elshof B, et al. The canine bilateral groove model of osteoarthritis. J Orthop Res 2008;26:14717.

15. Frisbie DD, Cross MW, Mcllwraith CW. A comparative study of articular cartilage thickness in the stifle of animal species used in human pre-clinical studies compared to articular cartilage thickness in the human knee. Vet Comp Orthop Traumatol 2006;19:142-6.

16. van Valburg AA, Wenting MJ, Beekman B, et al. Degenerated human articular cartilage at autopsy represents preclinical osteoarthritic cartilage: comparison with clinically defined osteoarthritic cartilage. J Rheumatol 1997;24:358-64.

17. Cook J, Kuroki K, Visco DM, et al. The OARSI histopathology initiative - recommendations for histological assessments of osteoarthritis in the dog. Osteoarthritis and cartilage/OARS, Osteoarthritis Research Society 2010;18: S66-S79.

18. Lafeber FP, Vander Kraan PM, Van Roy JL, et al. Articular cartilage explant culture; an appropriate in vitro system to compare osteoarthritic and normal human cartilage. Connect Tissue Res 1993;29:287-99.

19. Stockwell RA. The interrelationship of cell density and cartilage thickness in mammalian articular cartilage. J Anat 1971; 109:411-21.

20. Hunziker EB, Quinn TM, Hauselmann HJ. Quantitative structural organization of normal adult human articular cartilage. Osteoarthritis and cartilage/OARS, Osteoarthritis Research Society. 2002;10: 564-72.

21. Schulze-Tanzil G, Muller RD, Kohl B, et al. Differing in vitro biology of equine, ovine, porcine and human articular chondrocytes derived from the knee joint: an immunomorphological study. Histochem Cell Biol 2009;131:219-29.

22. Martin JA, Buckwalter JA. Human chondrocyte senescence and osteoarthritis. Biorheology 2002;39:145-52.

23. Brandt KD. An atlas of osteoarthritis. The Parthenon publishing group 2001.

24. Frost-Christensen LN, Mastbergen SC, Vianen ME, et al. Degeneration, inflammation, regeneration, and pain/disability in dogs following destabilization or articular cartilage grooving of the stifle joint. Osteoarthritis and cartilage/OARS, Osteoarthritis Research Society 2008;16: 1327-35. 